\title{
Risk factors of multiple sclerosis in Aseer region, Kingdom of Saudi Arabia
}

\author{
A case-control study
}

Aesha F. Siddiqui, DFM, MD, Abdullah A. Alsabaani, MPH, PhD, Abmed Y. Abouelyazid, PhD, Yasser I. Wassel, MD.

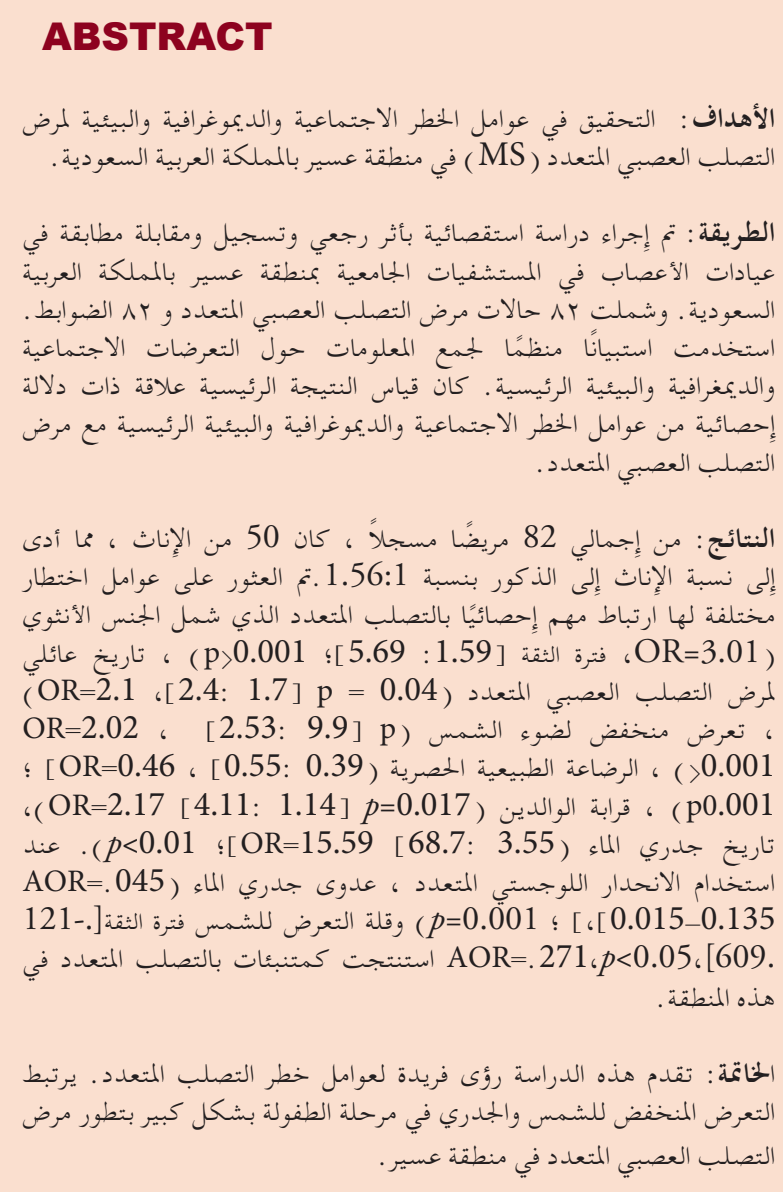

Objectives: To investigate socio-demographic and environmental risk factors of multiple sclerosis (MS) in Aseer region, Kingdom of Saudi Arabia (KSA).

Methods: This was a retrospective, record, and interview based matched case control study completed in the neurology clinics at tertiary hospitals in Aseer, KSA. It included 82 MS cases and 82 controls. The study used a structured questionnaire to collect information on key socio-demographic and environmental exposures. The main outcome measure was a statistically significant relationship of key sociodemographic and environmental risk factors with MS.

Results: A total of 82 registered patients, 50 were females, resulting in a female to male ratio of $1.56: 1$. Various risk factors were found to have a statistically significant association with MS which included female $(\mathrm{OR}=3.01$, 95\% CI [1.59:5.69]; $p<0.001)$, family history of MS $(\mathrm{OR}=2.1,95 \% \mathrm{CI}$ [2.4:1.7] $p=0.04)$, low exposure to sunlight $(\mathrm{OR}=2.02$, 95\%CI [2.53: 9.9] $p<0.001)$, only fed breast milk in childhood $(\mathrm{OR}=0.46,95 \% \mathrm{CI}$ [0.55:0.39]; $p<0.001)$, parental consanguinity ( $\mathrm{OR}=2.17$ 95\%CI [4.11:1.14] $p=0.017$ ), history of chickenpox $(\mathrm{OR}=15.59$ 95\% CI [68.7:3.55]; $p<0.01)$. On using multiple logistic regression, chicken pox infection (AOR $=0.045$, 95\%CI $[0.015-0.135] ; p=0.001$ ) and low sun-exposure $(\mathrm{AOR}=.271,95 \% \mathrm{CI}[.121-.609] ; p<0.05)$ were deduced as the predictors of MS in this region.

Conclusion: This study offers unique insights into the risk factors of MS. Low sun exposure and childhood chickenpox are significantly related to the development of MS in the Aseer region.

$$
\begin{aligned}
& \text { Neurosciences 2021; Vol. } 26 \text { (1): 69-76 } \\
& \text { doi: 10.17712/nsj.2021.1.20200107 }
\end{aligned}
$$

From the Department of Family and Community Medicine (Siddiqui), from the Department of Family and Community Medicine (Alsabaani), King Khalid University, Abha; from the Department of Preventive Medicine (Abouelyazid), from the Department of Neurology (Wassel), Armed Forced Hospital Southern Region, Khamis Mushayt, Kingdom of Saudi Arabia; and from the Department of Public Health (Abouelyazid, Wassel), Faculty of Medicine, Mansoura University, Egypt.

Received 1st July 2020. Accepted 21st September 2020.

Address correspondence and reprint request to: Dr. Aesha F. Siddiqui, Assistant Professor, Family and Community Medicine, King Khalid University, Abha, Kingdom of Saudi Arabia. E-mail:draeshasiddiqui@gmail.com

ORCID ID: https://orcid.org/0000-0003-1026-6438 
$\mathrm{M}$ ultiple sclerosis (MS) is disease affecting the central nervous system, that mainly occurs in young people and often incapacitates them for life. Autoimmunity along with several environmental risk factors plays an important role in its etiology, however the precise pathogenic mechanisms that bring on the development of MS have yet to be clearly identified. ${ }^{1-4}$ Multiple sclerosis, like other autoimmune diseases, is hypothesized to result from complex interactions between genetic susceptibility and environmental factors. ${ }^{5}$

Environmental factors with established risk of developing MS include low sunlight exposure, vitamin D insufficiency, and smoking. ${ }^{3,6}$ Environmental effects on the development of MS are strongly suggested by its geographic distribution. The prevalence rates of MS are highest in regions at high latitudes and low in regions near the equator. ${ }^{1}$ The effect of latitude acts largely through exposure to sunlight and vitamin D synthesis in the body. Evidence of changes in MS risk after migration supports the influence of environmental factors on disease susceptibility. ${ }^{7}$

Both active and passive cigarette smoking and MS risk have demonstrated a dose-dependent relationship. Increased MS risk is associated with cumulative smoking, which is reflected by serum nicotine levels. This risk typically subsides after a decade of smoking cessation. ${ }^{8}$ Case control studies have shown an estimated $40-80 \%$ increased risk of developing MS associated with smoking. ${ }^{9}$ Another risk factor that can induce or promote autoimmunity is microbial infection, ${ }^{10,11}$ which accelerate the autoimmune processes. Chicken pox or varicella zoster virus (VZV), which is a neurotropic virus that remains in the nervous system for decades, has been hypothesized to be a potential microbial agent in the pathogenesis of MS. ${ }^{12} \mathrm{~A}$ recent review supports the hypothesis and mentions several previous studies that have reported VZV DNA in the blood lymphocytes and cerebrospinal fluid (CSF) of MS patients during acute relapses, disappearing during phases of remission. ${ }^{13}$ Though these findings support the direct participation of $\mathrm{VZV}$ in the etiology of MS, however, conclusive evidence needs to be established. The association between breastfeeding and MS has also been investigated in a few studies, in which prolonged breastfeeding has shown a protective effect. ${ }^{14,15}$

Disclosure. Authors have no conflict of interests, and the work was not supported or funded by any drug company.
The incidence of MS has amplified quickly worldwide. ${ }^{16,17}$ In particular, this increase has affected the countries of the Arabian Gulf. ${ }^{18,19}$ Observing this quick rise in prevalence of MS suggests that environmental factors have played a more important role in this change than genetic factors. Unlike genetic risk factors, environmental and lifestyle factors are modifiable, and most regions around the globe, especially the Gulf countries, have shown remarkable socioeconomic and lifestyle changes in the past few decades. ${ }^{17}$ Most epidemiological studies focusing on the impact of environmental exposures on MS have occurred in the West. ${ }^{20}$ Given the reported increase in the incidence of MS both globally and in the Middle East, there is an urgent need to understand the role of modifiable factors traditionally implicated in the causation of MS. Information regarding these factors, which could play a role in disease causation as well as protection, could be incorporated into patient care and preventive programs, particularly for individuals with positive family histories of MS and therefore increased risk of developing the disease. The present paper presents data from a study of the distribution of disease characteristics and risk factors of MS in Southwest Saudi Arabia.

Methods. A retrospective, record and interviewbased, and matched case control study included patients with MS attending neurology clinics at the Armed Forces Hospitals Southern Region and Aseer Central Hospital Abha, Saudi Arabia between April 2017 and April 2018. There were 105 total registered cases $(58$ registered cases at the neurology clinic of Armed Forces Hospital, Khamis Mushayt and 47 cases at Aseer central hospital, Abha). A total of 82 cases formed the final sample for case group. For the purposes of this study, cases were defined as patients aged 18 years or older, previously diagnosed with MS or clinically isolated syndrome (CIS) by a neurologist, and regularly attending the neurology clinic of one of the 2 aforementioned hospitals. Patients aged less than 18 years and those who refused to participate were excluded. Single controls matched for age and region were selected from among the relatives of the patients and from outpatient clinics.

For the purpose of data collection, we developed a structured questionnaire based on previous studies of MS and its risk factors. ${ }^{21-24}$ For searching previous studies and review of literature, search was done using Google scholar, Pubmed, and Saudi Digital Library. Information collected with this questionnaire included details regarding: i) basic patient characteristics, such as age, gender, height, weight, and BMI; ii) socioeconomic 
factors, including education, occupation, residence, and handedness; iii) environmental factors, such as smoking, sunlight exposure, and exposure to animals; iv) medical factors, such as family history of MS, parental consanguinity, history of vitamin D deficiency, comorbidity with an autoimmune disease (such as, type 1 diabetes, psoriasis, ankylosing spondylitis, celiac disease, hypothyroidism, or hyperthyroidism), and history of hospital admission for an infection (such as pneumonia, UTI, meningitis, or GI infection) or for surgical trauma; and v) childhood factors, including history of breastfeeding, vaccination, and childhood infections, such as measles, chicken pox, rubella, and mumps. This questionnaire was also used to record information regarding disease characteristics described in a previous paper. ${ }^{25}$

Statistical analysis. Data was collected from patients with an interview questionnaire. The interviews took place in neurology clinics on the participants' scheduled days of clinic appointments. Medical records were used to gather any missing responses or data that the patients could not provide (drug names and disease types). Data was analyzed using the Statistical Package for Social Sciences, version 23 (IBM Corp, Armonk, NY, USA). Descriptive statistics were used to illustrate the cases and controls. Frequencies and percentages were used to describe the demographic and disease characteristics of the cases (described in another paper). ${ }^{25} \mathrm{~A}$ univariate analysis was conducted using a Chi-square test and a Fisher's exact test to study the association of various independent probable risk factors with MS. The odds ratio (OR) and $95 \%$ confidence interval (CI) associated with each potential risk factor were calculated as well. To test for interactions among the variables, a multiple logistic regression analysis was applied in a backward stepwise model using MS as the dependent variable and using socioeconomic, environmental, and familial variables as the independent variables. The $p$ values $<0.05$ were considered significant.

The study was conducted upon approval by the institutional ethics committee [AFHSRMREC/2017/ COMMUNITY MED/229]. In accordance with the principles of the Helsinki Declaration, all participants were provided with detailed information regarding the study prior to enrollment. Anonymity and confidentiality of participant information were assured, and consent was obtained from all participants.

Results. The results of this study are described in following sequence: socio-demographic variables, environmental variables, family history and autoimmune disease variables, and childhood history variables from
Tables 1-4. The final table (Table 5) presents results of multilogistic regression analysis.

Table 1 presents detailed information on the association of socio-demographic factors with MS cases. Comparison between the cases and controls revealed no significant difference regarding age, income or marital status. There was significantly higher number of females among cases as compared to controls (OR 3.01, 95\% CI [1.59-5.69]; $p=0.001)$. Left handedness was found more commonly among the control group.

Table 2 shows comparison between the cases and controls regarding potential risk factors in the environment revealed that $78 \%$ of those reporting a low exposure to sunlight are in the case group as compared to $41.5 \%$ in the control group. The odds of having MS are more than twice in the group with low exposure to sunlight. This difference was found to be statistically significant with (OR 2.02 95\% CI [2.53-9.9]; $p<0.0001$ ). Cigarette smoking was twice as common among controls (OR 2.68 95\% CI [1.27-5.52]; $p<0.001$ ). Comparison between the cases and controls regarding exposure to animals revealed that the control group had increased exposure to cats. However, there was no significant difference between the 2 groups regarding exposure to any type of animal.

Table 3 depicts the relationship of familial history and autoimmune disorders with MS. History of consanguinity, example marriage between relatives revealed that as compared to controls, more cases of MS showed positive history of consanguinity among their parents. This finding was statistically significant. Parental consanguinity poses twice the risk of MS as compared to no parental consanguinity (OR 2.17 95\% CI [1.14-4.11]; $p<0.017)$. Regarding family history of MS, and previous diagnosis of Vitamin D deficiency, both these risk factors were higher among cases. Among cases, approximately $5 \%$ had a positive family history of MS as compared to none in controls. Among cases, $61 \%$ reported a previous diagnosis of vitamin $D$ deficiency as compared to controls (56.1\%). However the difference was not statistically significant for these factors. The presence of other autoimmune disease as a risk factor for MS was also presented. Type 1 diabetes is significantly more among cases $(12.2 \%)$ as compared to controls(2.4\%), (OR 5.56, 95\% CI [1.18-26.2] $p=0.03$ ). Psoriasis is 3 times more common with MS as compared to controls, $(\mathrm{OR}=3.02$, 95\% CI [0.92-9.92]; $p=0.01)$. Celiac disease was significantly higher among MS cases $(\mathrm{OR}=12.5,95 \% \mathrm{CI}[1.58-99.6] ; p=0.003)$ as compared to control group.

Table 4 shows the association of childhood risk factors with MS. The percentage of respondents who were only breast fed was significantly higher among 
Risk factors of multiple sclerosis ... Siddiqui et al

Table 1 - Relationship of socio-demographic factors with multiple sclerosis.

\begin{tabular}{lccccc}
\hline Risk factor & Cases & Control & $\chi 2$ & OR $(\mathrm{CI})$ & $P$-value \\
\hline $\begin{array}{l}\text { Gender } \\
\quad \text { Male }\end{array}$ & $32(39.0)$ & $54(65.9)$ & & & \\
$\quad \begin{array}{l}\text { Female } \\
\text { Body mass index }\end{array}$ & $50(61.0)$ & $28(34.1)$ & 11.8 & $3.01(1.59: 5.69)$ & 0.001 \\
$\quad$ Non-obese & $39(47.5)$ & $35(42.6)$ & & & \\
$\quad \begin{array}{l}\text { Obese } \\
\text { Marital status }\end{array}$ & $43(52.5)$ & $47(57.4)$ & 0.394 & $0.82(0.44: 1.52)$ & 0.319 \\
$\quad$ Married & $58(70.7)$ & $48(58.5)$ & & & \\
$\quad$ Single & $24(29.3)$ & $34(41.5)$ & 2.66 & $0.58(0.30: 1.11)$ & 0.07 \\
$\begin{array}{l}\text { Left handedness } \\
\text { Yes }\end{array}$ & $4(4.9)$ & $20(24.4)$ & $*$ & 6.29 & \\
$\quad$ No & $78(95.1)$ & $62(75.6)$ & & {$[2.04: 19.35]$} & $<0.05$ \\
\hline
\end{tabular}

Values are presented as numbers and percentages $(\%)$. ${ }^{*}$ Fisher's exact test, CI - confidence interval, OR: odds ratio

Table 2 - Association of environmental risk factors with multiple sclerosis.

\begin{tabular}{lccccc}
\hline Variables & Cases & Control & $\chi^{2}$ & OR [95\% CI] & $P$-value \\
\hline $\begin{array}{l}\text { Low sunlight exposure } \\
\begin{array}{l}(<15 \text { min/ day }) \\
\text { Yes }\end{array}\end{array}$ & $64(78.0)$ & $34(41.5)$ & & & \\
$\quad$ No & $18(22.0)$ & $48(58.5)$ & 22.8 & $2.02[2.53: 9.9]$ & 0.000 \\
$\begin{array}{l}\text { Cigarette Smoking } \\
\text { Yes }\end{array}$ & $14(17.1)$ & $29(35.4)$ & & & \\
No & $68(82.9)$ & $53(64.6)$ & 7.1 & $2.68[1.27: 5.52]$ & 0.008 \\
$\begin{array}{l}\text { Animal exposure } \\
\text { Yes }\end{array}$ & $4(4.9)$ & $3(3.7)$ & & \\
No & $78(95.1)$ & $79(96.3)$ & $*$ & $1.3[0.29: 6.2]$ & 0.699 \\
\hline \multicolumn{5}{c}{$*$ Fisher's exact test, OR - odds ratio } \\
\hline
\end{tabular}

Table 3 - Relationship of family history and autoimmune diseases with multiple sclerosis.

\begin{tabular}{|c|c|c|c|c|c|}
\hline Risk Factor & Cases & Controls & $\chi^{2}$ & OR $[95 \% \mathrm{CI}]$ & $P$-value \\
\hline \multicolumn{6}{|c|}{ Parental consanguinity } \\
\hline Yes & $40(48.8)$ & $25(30.5)$ & \multirow{2}{*}{5.734} & \multirow{2}{*}{2.17 [1.14: 4.11] } & \multirow{2}{*}{0.017} \\
\hline No & $42(51.2)$ & $57(69.5)$ & & & \\
\hline \multicolumn{6}{|c|}{ Family history } \\
\hline Yes & $4 \quad(4.9)$ & $0 \quad(0.0)$ & \multirow{2}{*}{ * } & \multirow{2}{*}{$2.1[1.7: 2.4]$} & \multirow{2}{*}{0.06} \\
\hline No & $78(95.1)$ & $82(100)$ & & & \\
\hline \multicolumn{6}{|c|}{$\begin{array}{l}\text { Previous Vitamin D } \\
\text { deficiency }\end{array}$} \\
\hline Yes & $50(61.0)$ & $46(56.1)$ & \multirow{2}{*}{0.4} & \multirow{2}{*}{$1.2[0.65: 2.27]$} & \multirow{2}{*}{0.52} \\
\hline No & $32(39.0)$ & $36(43.9)$ & & & \\
\hline \multicolumn{6}{|c|}{ Autoimmune disease } \\
\hline \multicolumn{6}{|c|}{ Type 1 Diabetes } \\
\hline Yes & $10(12.2)$ & $2(2.4)$ & \multirow{2}{*}{ * } & \multirow{2}{*}{5.56 [1.18: 26.2] } & \multirow{2}{*}{0.03} \\
\hline No & $72(87.8)$ & 80 (97.6) & & & \\
\hline \multicolumn{6}{|l|}{ Psoriasis } \\
\hline Yes & $11(13.4)$ & $4(4.9)$ & \multirow[t]{2}{*}{ * } & \multirow{2}{*}{3.02 [0.92: 9.92$]$} & \multirow{2}{*}{0.01} \\
\hline No & $71(86.6)$ & $87(95.1)$ & & & \\
\hline \multicolumn{6}{|c|}{ Celiac disease } \\
\hline Yes & $11(13.4)$ & $1 \quad(1.2)$ & \multirow{2}{*}{ * } & \multirow{2}{*}{12.5 [1.58: 99.6] } & \multirow{2}{*}{0.003} \\
\hline No & $71(86.6)$ & $81(98.8)$ & & & \\
\hline & & er's exact test & $\mathrm{R}$ - odc & & \\
\hline
\end{tabular}


Table 4 - Association of childhood risk factors with multiple sclerosis.

\begin{tabular}{lccccc}
\hline Childhood factor & Cases & Controls & $\chi^{2}$ & P-value & $\begin{array}{c}\text { Odds ratio } \\
{[95 \% \mathrm{CI}]}\end{array}$ \\
\hline $\begin{array}{l}\text { Only fed breast milk } \\
\text { Yes }\end{array}$ & $72(87.8)$ & $82(100)$ & $*$ & 0.001 & $0.46[0.39: 0.55]$ \\
$\quad$ No & $10(13.0)$ & $0(0.0)$ & & & \\
$\begin{array}{l}\text { Complete childhood vaccination } \\
\text { Yes }\end{array}$ & $56(71.8)$ & $34(41.5)$ & 14.9 & 0.000 & $3.59[1.85: 6.95]$ \\
No & $22(28.2)$ & $48(58.5)$ & & & \\
$\begin{array}{l}\text { Chicken pox } \\
\text { Yes }\end{array}$ & $23(28.0)$ & $2(2.4)$ & $*$ & $<0.01$ & $15.59[3.55: 68.7]$ \\
No & $59(72.0)$ & $80(97.6)$ & & \\
\hline & *Fisher's exact test \\
\hline
\end{tabular}

Table 5 - Multiple logistic regression analysis for risk factors of multiple sclerosis.

\begin{tabular}{|c|c|c|c|c|c|c|}
\hline \multirow{2}{*}{ Variables } & \multirow{2}{*}{ Cases } & \multirow{2}{*}{ Controls } & \multirow{2}{*}{ Sig. } & \multirow{2}{*}{ AOR } & \multicolumn{2}{|c|}{$95 \% \mathrm{CI}$} \\
\hline & & & & & Lower & Upper \\
\hline \multicolumn{7}{|c|}{$\begin{array}{l}\text { Chicken pox } \\
\text { infection in childhood }\end{array}$} \\
\hline Yes & $23(28.0)$ & $2(2.4)$ & \multirow{2}{*}{$<0.001$} & \multirow{2}{*}{0.045} & \multicolumn{2}{|c|}{ Reference } \\
\hline No & $59(72.0)$ & $80(97.6)$ & & & 0.015 & 0.135 \\
\hline \multicolumn{7}{|c|}{ Sun exposure $<15$ mins. } \\
\hline Yes & $64(78.0)$ & $34(41.5)$ & \multirow{2}{*}{$<0.05$} & & \multicolumn{2}{|c|}{ Reference } \\
\hline No & $18(22.0)$ & $48(58.5)$ & & 0.271 & 0.121 & 0.609 \\
\hline
\end{tabular}

the control group. All the controls reported history of breastfeeding in childhood, while $87.8 \%$ cases reported the same and it showed a protective effect against MS $(\mathrm{OR}=0.46,95 \%$ CI [0.39-0.55]; $p=0.001)$. Complete childhood vaccination was significantly more evident in the case group (71\%). The history of viral infection especially as regard; measles, rubella and mumps shows no significant difference but chicken pox gives highly significant higher rates among cases. More than half of the cases reported history of chicken pox as compared to less than 5\% among the controls (OR 15.5 , 95\% CI [3.55-68.7]; $p<0.01)$.

Table 5 presents the significant results from the multivariate logistic regression analysis. This was performed using forward stepwise selection to find the predictors of MS. Only those variables that were found significant in univariate analysis were entered into the logistic model. Chicken pox exposure in childhood, and poor exposure to sunlight were obtained as predictors of MS risk.

Discussion. This study provided information regarding the sociodemographic, environmental, and childhood risk factors of MS, including history of parental consanguinity, sun exposure, vitamin $\mathrm{D}$ deficiency, smoking, childhood infections, and breastfeeding in the Aseer region of Saudi Arabia.

Comparisons between the cases and the controls revealed no significant differences in sociodemographic factors such as age, income, or marital status. There was a significantly higher number of female participants among the cases than among the controls. Studies from around the globe have reported a female preponderance of MS. ${ }^{26,27}$ The gender gradient in this study is confirmed from the Saudi registry of MS, which has established a 2:1 female:male ratio of MS in the country. ${ }^{28}$ This difference could be explained by inherent differences in genetics and in physiological responses to other proposed risk factors, such as environmental and medical risk factors. It has also been proposed that the female preponderance of MS could be related to changes in environmental interactions rather than genetic factors. ${ }^{27}$

One important finding of the present study was the significant association between MS and parental consanguinity in univariate analysis. This association was explored previously in a study in Saudi Arabia, which failed to establish any such relationship ${ }^{29}$ and 
reported that while positive family history of MS was more common among the cases than among the controls, this association was not statistically significant. This finding is particularly important in regions wherein consanguinity is a common occurrence and needs further evaluation.

Among the suggested environmental risk factors of MS, low sunlight exposure and vitamin D deficiency have occupied an especially important place in the literature. In the present study, comparison between the cases and the controls revealed that the odds of having MS were more than doubled in the group that reported low exposure to sunlight. This difference was found to be statistically significant. Many studies have focused on the role of low sun exposure and vitamin D as risk factors and management for MS. ${ }^{30,31}$ It is known that vitamin $\mathrm{D}$ synthesis is dependent on adequate sun exposure. The full body covering practiced by females in many middle eastern countries may be related to the higher rate of vitamin D deficiency observed in these populations and may contribute to the higher rate of MS in female population and in those who report lower sun exposure in these populations. ${ }^{32,33}$ Lower sun exposure could also be caused by is the reduced physical activity levels of Middle Eastern populations, consequent to lifestyle changes caused by the rise of industrialization and urbanization. ${ }^{34}$

One of the important risk factors addressed in this study was cigarette smoking, which was twice as common among controls. This was an interesting finding, as it was contrary to the findings of other studies, however it can be attributed to the larger male sample among control group. Smoking has been identified as a major cause of many chronic diseases and an established risk factor for developing MS. ${ }^{28,35}$ In fact, considerable evidence implicates smoking to be an independent risk factor for MS. ${ }^{3,4,6}$ The absence of such a relationship in the present study could be attributed to the study sample and the degree of variation seen in the effects of smoking on the immune system. The effects of confounding variables should be considered as well. Further studies are needed to determine whether smoking affects the progression of MS in Saudi population, as has been observed in Western populations. ${ }^{6}$

Comparison between the cases and controls in terms of exposure to animals revealed that the control group reported increased exposure to cats. However, there were no significant differences between the 2 groups in terms of exposure to any type of animal. This finding was similar to that of a study in Iran, which did not reveal any association between previous exposure to farm animals or pets and risk of developing MS. ${ }^{31}$
The investigation of other autoimmune diseases as potential risk factors for MS showed that type 1 diabetes, psoriasis, celiac disease, ankylosing spondylitis, and hyperthyroidism were more common among cases of MS; of these associations, only those of psoriasis and celiac disease were statistically significant. It has been reported that the prevalence of autoimmune conditions is highest in Western countries; and may indicate a correlation with Western lifestyle. ${ }^{36}$ Some studies suggest that higher standards of living lead to insufficient exposure to foreign agents and consequently the immune system is poorly adapted. ${ }^{3,4}$ Thus, individuals with higher standards of living are at higher risk of developing autoimmune diseases, such as MS. ${ }^{3,4}$ Residents of the Middle East lead lifestyles similar to those of Western countries owing to economic developments and rising urbanization, and this is contributing to rising lifestyle related diseases in the region. ${ }^{37}$

Feeding with breast milk in childhood has previously been identified as a protective factor for many diseases, including bronchial asthma, atopic dermatitis, type 1 diabetes mellitus, and Crohn's disease through its immunogenic effects. ${ }^{38}$ In the present study, childhood history of feeding with breast milk appeared to have a protective effect against MS. This finding was in concordance with those of previous studies, which have shown an association between prolonged feeding with breast milk and decreased risk of MS in children. ${ }^{14} \mathrm{~A}$ German study suggested that only breastfeeding for more than 4 months, rather than breastfeeding in general, has a protective effect against MS. ${ }^{15}$ However, the duration of breastfeeding was not inquired in the present study.

Other childhood influences considered in this study included complete vaccination and history of viral infections, particularly measles, rubella, mumps, and chicken pox. Out of all these factors, only a history of chicken pox in childhood was reported significantly more by the cases than by the controls. Childhood chicken pox was also one of the 2 identified predictors of MS, along with low sunlight exposure. Previous studies have postulated that VZV may be a factor in the pathogenesis of MS, as a progressive increase in the incidence of MS has been linked to childhood chicken pox. ${ }^{11,20}$

Study limitations. This study was limited by its small sample size. Though we covered the 2 main hospitals run by the ministry of health, we had only 82 cases (out of 105 registered) who agreed to participate in the study. We selected a random sample of controls, matching only for age and region but not for gender as we wanted 
to study the gender gradient in our sample. Due to this limitation, the findings cannot be generalized to all of Saudi Arabia. Another problem associated with this study is that of a retrospective design where information is based on the patients memory. Patients were required to provide information from their past, including childhood and this contributes to recall bias.

In conclusion, the present study offers unique insights into the risk factors of MS in the Aseer region of Saudi Arabia. The results of this study indicated that low sun exposure and childhood chicken pox are risk factors for the development of MS in the Aseer region of Saudi Arabia. With the increasing number of people affected by MS, future prospective studies are recommended to focus on these risk factors.

Acknowledgment. The authors gratefully acknowledge Scribendi (www.scribendi.com) for English language editing. Special gratitude to Dr Mohammad Saad, Ministry of Health, and Professor Shamsun Nahar, Department of Family and Community Medicine, King Khalid University for their support.

\section{References}

1. Dendrou CA, Fugger L, Friese MA. Immunopathology of multiple sclerosis. 20; Nat Rev Immunol 2015; 15: 545-558.

2. Sakoda A, Matsushita T, Nakamura Y, Watanabe M, Shinoda K, Masaki K, et al. Environmental risk factors for multiple sclerosis in Japanese people. Mult Scler Relat Disord 2019; 38: 101872.

3. Marrodan M, Alessandro L, Farez MF, Correale J. The role of infections in multiple sclerosis. Mult Scler J 2019; 25: 891-901.

4. Alfredsson L, Olsson T. Lifestyle and Environmental Factors in Multiple Sclerosis. Cold Spring Harb Perspect Med 2019; 9: a028944.

5. Patsopoulos NA. Genetics of multiple sclerosis: an overview and new directions. Cold Spring Harb Perspect Med 2018; 8: a028951.

6. Degelman M.L, Herman K.M. Smoking and multiple sclerosis: a systematic review and meta-analysis using the Bradford Hill criteria for causation. Mult Scler Relat Disord 2017; 17: 207-216.

7. Berg HP, Celius EG. Socioeconomic factors and immigrant population studies of multiple sclerosis. Acta Neurol Scand 2015; 132: 37-41.

8. Hedström A.K, Olsson T, Alfredsson L, Smoking is a major preventable risk factor for multiple sclerosis. Mult Scler 2016; 22: 1021-1026.

9. Poorolajal J, Bahrami M, Karami M, Hooshmand E. Effect of smoking on multiple sclerosis: a meta-analysis. J Public Health (Oxf) 2017; 39: 312-320.

10. Bar-Or A, Pender MP, Khanna R, Steinman L, Hartung HP, Maniar T, Epstein-Barr virus in multiple sclerosis: theory and emerging immunotherapies. Trends Mol Med 2020; 26: 296310.

11. Venkatesan A, Johnson RT. Infections and multiple sclerosis. Handb Clin Neurol 2014; 122: 151-171.

12. Kattimani Y, Veerappa AM. Complex interaction between mutant HNRNPA1 and $\mathrm{gE}$ of varicella zoster virus in pathogenesis of multiple sclerosis. Autoimmunity 2018; 51: 147-151.
13. Cavallo, S. Immune-mediated genesis of multiple sclerosis. $J$ Transl Autoimmun 2020; 3: 100039.

14. Brenton JN, Engel CE, Sohn MW, Goldman MD. Breastfeeding during infancy is associated with a lower future risk of pediatric multiple sclerosis. Pediatr Neurol 2017; 77: 67-72.

15. Conradi S, Malzahn U, Paul F, Quill S, Harms L, Bergh FT, et al. Breastfeeding is associated with lower risk for multiple sclerosis. Mult Scler 2013; 19: 553-558.

16. Browne P, Chandraratna D, Angood C, Tremlett H, Baker C, Taylor BV, et al. Atlas of multiple sclerosis 2013: a growing global problem with widespread inequity. Neurology 2014; 83: 1022-1024.

17. Sahraian MA, Sahebkar M, Dehghani R, Derakhshan-Jazari M, Kazami-Moghaddam V, Kouchaki E. Multiple sclerosis- A disease on a dramatically rising trend in Iran: Review of possible reasons. Iran J Neurol 2017; 16: 34-40.

18. Deleu D, Mir D, Al Tabouki A, Mesraoua R, Mesraoua B, Akhtar N, et al. Prevalence, demographics and clinical characteristics of multiple sclerosis in Qatar. Mult Scler 2013; 19: 816-819.

19. Alroughani R, Ahmed SF, Behbahani R, Khan R, Thussu A, Alexander KJ, et al. Increasing prevalence and incidence rates of multiple sclerosis in Kuwait. Mult Scler 2014; 20: 543-547.

20. Correale J, Farez MF, Inés M. Environmental factors influencing multiple sclerosis in Latin America. Mult Scler J-Exp Transl Clin 2017; 3: 2055217317715049.

21. Al-Shammri SN, Hanna MG, Chattopadhyay A, Akanji AO. Sociocultural and demographic risk factors for the development of multiple sclerosis in Kuwait: a case-control study. PLoS One 2015; 10: e0132106.

22. Gustavsen MW, Page CM, Moen SM, Bjolgerud A, Berg-Hansen P, Nygaard GO, et al. Environmental exposures and the risk of multiple sclerosis investigated in a Norwegian case-control study. BMC Neurol 2014; 14: 196.

23. Poorolajal J, Mazdeh M, Saatchi M, Talebi Ghane E, Biderafsh A, Lotfi B, et al. Multiple sclerosis associated risk factors: A casecontrol study. Iran J Public Health 2015; 44: 1498-1505.

24. El-Muzaini, H, Akhtar, S, Alroughani, R. A matched casecontrol study of risk factors associated with multiple sclerosis in Kuwait. BMC Neurol 2020; 20: 306.

25. Al-Abdullah MS, Siddiqui AF. Demographic and disease characteristics of multiple sclerosis in the Southwest Region of Saudi Arabia. Neurosciences (Riyadh) 2018; 23: 320-325.

26. Waubant E, Lucas R, Mowry E, Graves J, Olsson T, Alfredsson L, et al. Environmental and genetic risk factors for MS: an integrated review. Ann Clin Transl Neurol 2019; 6: 1905-1922.

27. Etemadifar M, Nourian SM, Nourian NS, Abtahi SH, Sayahi F, Saraf Z, et al. Early-onset multiple sclerosis in Isfahan, Iran: report of the demographic and clinical features of 221 patients. J Child Neurol 2016; 31: 932-937.

28. Al Jumah M, Bunyan R, Al Otaibi H, Al Towaijri G, Karim A, Al Malik Y, et al. Rising prevalence of multiple sclerosis in Saudi Arabia, a descriptive study. BMC Neurol 2020; 20: 49.

29. Al-Jumah M, Kojan S, Khathaami AA, Abdulkaream IA, Blawi MA, Jawhary A. Familial multiple sclerosis: does consanguinity have a role? Mult Scler J 2011; 17: 487-489.

30. Sintzel MB, Rametta M, Reder AT. Vitamin D and multiple sclerosis: a comprehensive review. Neurol Ther 2018; 7: 59-85. 
31. Jagannath VA, Filippini G, Di Pietrantonj C, Asokan GV, Robak EW, Whamond L, Robinson SA.Vitamin D for the management of multiple sclerosis. Cochrane Database Syst Rev 2018; 9: CD008422.

32. Al-Yatama FI, AlOtaibi F, Al-Bader MD, Al-Shoumer KA. The Effect of Clothing on Vitamin D Status, Bone Turnover Markers, and Bone Mineral Density in Young Kuwaiti Females. Int J Endocrinol 2019; 2019: 6794837.

33. Sampat N, Al-Balushi B, Al-Subhi L, Al-Adawi S, Essa MM, Walid Qoronfleh M. Vitamin D: Public Health Status Regional Gulf Region. Int J Nutr Pharmacol Neurol Dis 2019; 9: 117-135.

34. Moghadam VM. Urbanization and women's citizenship in the Middle East. Brown Journal of World Affairs 2010; 17: 19-34.
35. Rosso M, Chitnis T. Association between cigarette smoking and multiple sclerosis: a review. JAMA Neurol 2020; 77: 245-253.

36. Marrie RA, Reider N, Cohen J, Stuve O, Sorensen PS, Cutter $\mathrm{G}$, et al. A systematic review of the incidence and prevalence of autoimmune disease in multiple sclerosis. Mult Scler 2015; 21 : 282-293.

37. Azami M, YektaKooshali MH, Shohani M, Khorshidi A, Mahmudi L. Correction: Epidemiology of multiple sclerosisin Iran: A systematic review and meta-analysis. PLoS One 201; 14: e0219466.

38. Li C, Liu Y, Jiang Y, Xu N, Lei J. Immunomodulatory constituents of human breast milk and immunity from bronchiolitis. Ital J Pediatr 2017; 43: 8.

\section{Statistics}

Excerpts from the Uniform Requirements for Manuscripts Submitted to Biomedical Journals updated November 2003.

Available from www.icmje.org

Describe statistical methods with enough detail to enable a knowledgeable reader with access to the original data to verify the reported results. When possible, quantify findings and present them with appropriate indicators of measurement error or uncertainty (such as confidence intervals). Avoid relying solely on statistical hypothesis testing, such as the use of $P$ values, which fails to convey important information about effect size. References for the design of the study and statistical methods should be to standard works when possible (with pages stated). Define statistical terms, abbreviations, and most symbols. Specify the computer software used. 